\title{
Spatial coherence measurements in arrays of coupled vertical cavity surface emitting lasers
}

\author{
L. D. A. Lundeberg, a) G. P. Lousberg, D. L. Boiko, and E. Kapon \\ Laboratory of Physics of Nanostructures, Ecole Polytechnique Fédérale de Lausanne (EPFL), \\ Laboratory of Physics of Nanostructures, CH-1015 Lausanne, Switzerland
}

(Received 14 November 2006; accepted 11 December 2006; published online 8 January 2007)

\begin{abstract}
The spatial coherence and the optical phase distribution across a two-dimensional (2D) photonic crystal implemented with coupled arrays of vertical cavity surface emitting lasers (VCSELs) are experimentally characterized. This is achieved by performing Young's interference experiments between pairs of array elements using a spatial light modulator arrangement. In contrast to far-field measurements that provide information only on the global spatial coherence, this approach can yield full mapping of the complex degree of spatial coherence. Examples of such analysis are presented for nominally uniform one-dimensional and 2D arrays of coupled VCSELs and possible mechanisms of the observed coherence degradation are discussed. (C) 2007 American Institute of Physics. [DOI: 10.1063/1.2431474]
\end{abstract}

The output power of single-mode vertical cavity surface emitting lasers (VCSELs) is typically limited to a few milliwatts because of their small aperture area required for higher-order spatial mode suppression. ${ }^{1}$ Increasing the aperture size leads to poor selection between spatial modes and, above a certain size, results in uncontrolled filamentation, which limits the output power and degrades the spatial coherence and beam quality of the device. The problem of filamentation can be overcome by coupling a large number of single-mode VCSEL, forming two-dimensional (2D) arrays of phase-locked emitters. ${ }^{2-5}$ In addition, these arrays exhibit a high degree of spatial coherence, as inferred from their nearly diffraction limited, four-lobed far-field patterns, which indicate that they oscillate predominantly at the lowest loss out-of-phase supermode. ${ }^{2-5}$

Evaluating the features of spatial coherence of coupledVCSEL arrays is important not only for optimizing their coupling but also for developing functionalities that rely on such coherence. An example of such application is beam steering, where control over the mutual phase of the emitters is necessary.

The spatial coherence in phase-locked VCSEL arrays has traditionally been evaluated using measurements of their far-field patterns ${ }^{2,3}$ complemented by model calculations of their supermodes. ${ }^{4,5}$ However, this approach yields information only on the global coherence properties of the array, in particular, the deviation of the beam pattern from the expected diffraction limited distribution. Moreover, such analysis generally cannot give direct indications on the mechanisms of coherence degradation. Spectral analysis of such arrays, e.g., using spectrally resolved far-field patterns or spatially resolved emission spectra, could give more indications on spatial coherence, but is difficult due to the small spectral splitting of the supermodes in large arrays.

A more complete evaluation of the spatial coherence across a VCSEL array would be to measure the complex degree of spatial coherence $\gamma\left(x, y ; x^{\prime}, y^{\prime}\right)$ between pairs of points in the array plane $(x, y)$. This can be accomplished by performing Young's interference experiments, in which the

${ }^{a)}$ Electronic mail: lars.lundeberg@epfl.ch interference pattern corresponding to two selected points is measured and analyzed. Such analysis has been performed for one-dimensional (1D) arrays of phase-coupled, edge emitting diode lasers. ${ }^{6,7}$ In those experiments, an opaque screen with double slits was mechanically translated in one direction across the optical beam, and the interference fringe visibility was used to infer the degree of spatial coherence.

In this letter, we perform measurements of the degree of spatial coherence in a 2D coupled-VCSEL array, revealing details unattainable through standard far- and near-field measurements. We employ Young's interference measurements between pairs of array elements by using a configurable aperture generator based on a liquid crystal spatial light modulator. ${ }^{8}$ We observe a decay of the mutual coherence with increasing distance between array VCSELs, as well as deviations from perfect $\pi$ phase difference between adjacent emitters expected for the highest-order supermode. Possible origins of these coherence features are discussed.

The VCSEL arrays employed in this study were fabricated from wafers incorporating three strained InGaAs/AlGaAs quantum wells sandwiched between GaAs/AlGaAs distributed Bragg reflectors (DBRs), emitting at $943 \mathrm{~nm}$ wavelength. The bottom emitting, VCSEL arrays are defined by deposition of Au/Cr metallic overlays on the top DBR. The metallic overlays also serve as top electrical contact to provide uniform current injection into the active region of the entire VCSEL array. The individual VCSELs are defined by square Au pixels of high reflectivity, surrounded by a $\mathrm{Cr}$ grid of lower reflectivity. ${ }^{4}$ The $\mathrm{Au}$ pixels are $4.5 \mu \mathrm{m}$ wide, and the array square lattice period is $6 \mu \mathrm{m}$. Different 1D and 2D array designs were fabricated using proper photolithography masks. The structures were characterized at room temperature under pulsed current injection (typically, $50 \mathrm{~ns}$ pulses at $0.1 \%$ duty cycle).

Figures 1(a) and 1(b) depict the near- and far-field patterns of a $7 \times 7$ VCSEL array. The intensity nulls in between adjacent pixels in the near-field and the four-lobe far-field pattern indicate that nearest neighbor lattice sites have $\pi$ radians phase difference, which corresponds to the lowest $\operatorname{loss}^{9}$ out-of-phase supermode. ${ }^{10,11}$ The full width at half maximum (FWHM) of each major lobe is about $1.2^{\circ}$, which, 

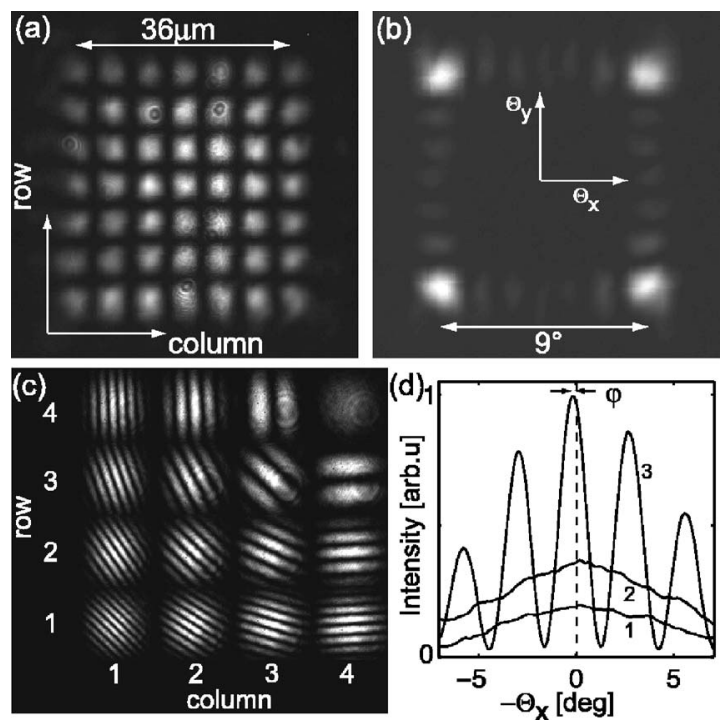

FIG. 1. (a) Near-field pattern of $7 \times 7$ VCSEL array and (b) its far-field pattern. (c) Interference patterns between the center VCSEL $[\{$ column, row $\}=\{4,4\}]$ and VCSELs in columns 1-4, rows 1-4. (d) Farfield line scans of solitary VCSELs $\{4,4\}$ and $\{1,4\}$ (curves 1 and 2 ) and their interference pattern measured perpendicular to the plane of the fringes (curve 3).

compared to the diffraction limited width of $1.14^{\circ}$, is a good indication of a global high degree of coherence across the lattice. This theoretical FWHM used, $0.886 \lambda / N \Lambda$ for $N \times N$ array, assumes that the intensity distribution across the array is constant. However, the measured near-field intensity pattern is slightly peaked at the center of the array, which reduces the effective spatial mode extent and thus broadens the far-field lobes. Since the far-field lobe width is dependent on the details of the modal distribution, more quantitative evaluation of the spatial coherence cannot be made based on these measurements alone.

The complex degree of spatial coherence $\gamma_{i j}$ between each pair of VCSELs in the array was evaluated by measuring the interference patterns between them using the scheme described in Ref. 8. The near-field pattern of the array was imaged on the spatial light modulator, which was programed to select the desired VCSEL pairs. Figure 1(c) shows several such interference patterns obtained by interfering the center VCSEL with other VCSELs of the $7 \times 7$ array. Note that the intensity at the center of the patterns alternates with VCSEL spacing between a null and a maximum, evidencing the general out-of-phase relationship between adjacent pixels for the lowest loss supermode.

The complex degree of coherence $\gamma_{i j}$ was extracted from scans of these interference patterns, measured perpendicular to the plane of the fringes. An example of such scan is shown in Fig. 1(d), where the diffraction patterns of the individual selected VCSELs by itself are also shown. The interference intensity pattern is related to the complex degree of spatial coherence by $I=I_{i}+I_{j}+2 \sqrt{I_{i} I_{j}}\left|\gamma_{i j}\right| \cos \left[\alpha_{i j}-\varphi\right]$, where $I_{i}$ and $I_{j}$ represent the measured far-field intensities for the individual VCSELs. The absolute value of $\gamma_{i j}$ is thus measured directly from the fringe pattern and the individual VCSEL intensities, whereas the phase $\varphi$ is extracted from the position of the fringes relative to the center of the far-field pattern $\left[\left(\theta_{x}, \theta_{y}\right)\right.$ $=(0,0)$, Fig. 1(b)].

The complex degree of spatial coherence for the array of Fig. 1, measured with respect to the center VCSEL, is shown Downloaded 10 Jan 2008 to 139.165.16.222. Redistribution subject
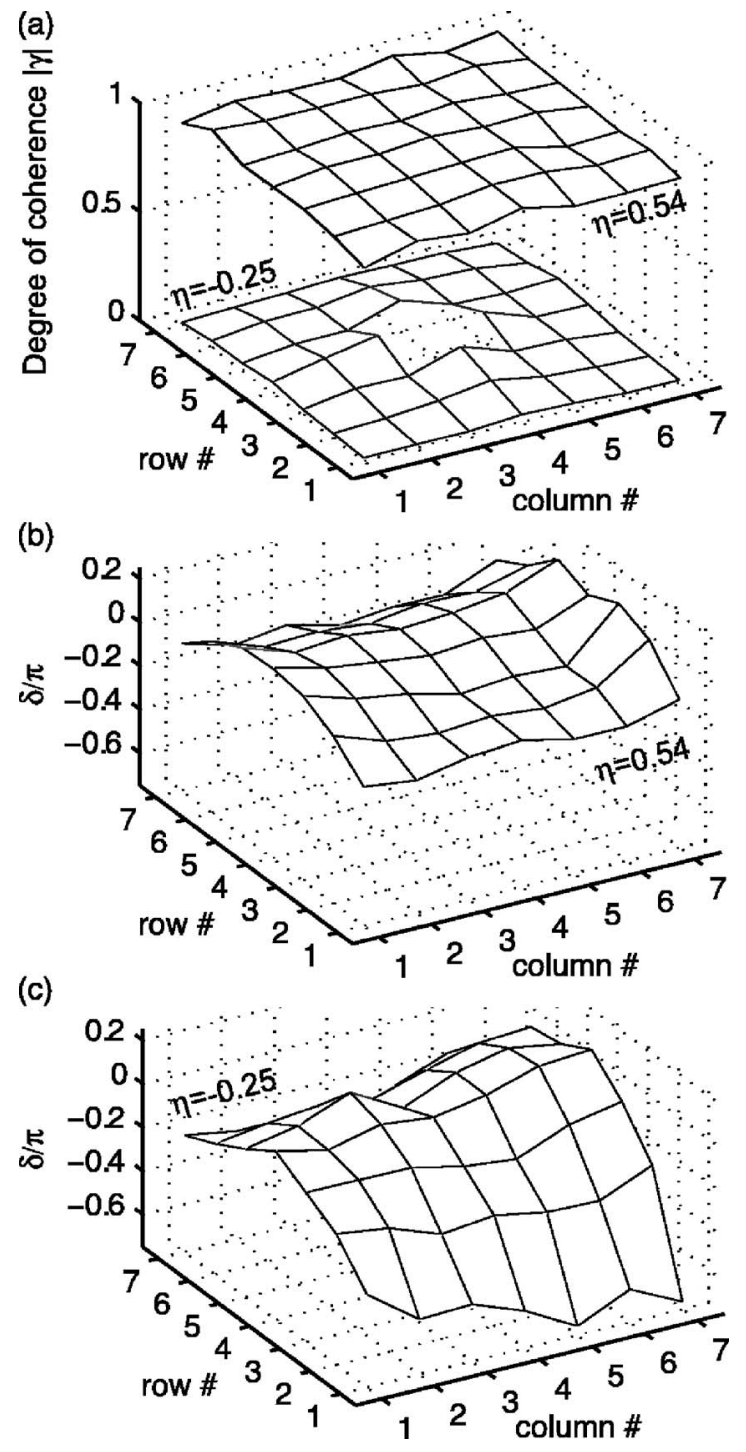

FIG. 2. (a) Degree of spatial coherence $\left|\gamma_{12}\right|$ of individual VCSELs of $7 \times 7$ array with respect to the array center VCSEL below $(\eta=-0.25)$ and above $(\eta=0.54)$ the lasing threshold. The degree of self-coherence for the center pixel has been left out below threshold and is put to $\left|\gamma_{11}\right|=1$ above threshold. Detuning from the out-of-phase relationship $\delta$ (b) above and (c) below the lasing threshold.

in Fig. 2 for different values of the relative excess above threshold $\eta=\left(I-I_{\mathrm{th}}\right) / I_{\mathrm{th}}$, both below $(\eta=-0.25)$ and above $(\eta=0.54)$ threshold. Above threshold $\left|\gamma_{i j}\right|$ is close to unity across the array, with an average value $|\gamma|=0.96$ at $\eta=0.54$ [see Fig. 2(a)]. However, a slight decay of this value towards the border of the array is noticeable. Below threshold, the degree of coherence is uniformly low across the array, with an average value of $|\gamma|=0.05$. The mutual coherence between the center pixel and its closest vertical and horizontal neighbors is noticeably higher than with the other pixels. Interestingly, the mutual coherence between the center pixel and the second nearest neighbors along the main lattice axes is higher than the mutual coherence between the center pixel and its nearest neighbors located along the lattice diagonals. This indicates that the effective coupling along the main lattice axes is stronger than along the lattice diagonals.

Figures 2(b) and 2(c) depict the phase detuning $\delta$ from the expected $\pi$ phase difference $(\delta=\varphi$ for nominally inphase pixels and $\delta=\varphi-\pi$ for nominally out-of-phase pixels) between adjacent pixels to the reference center pixel, above
to AIP license or copyright; see http://apl.aip.org/apl/copyright.jsp 


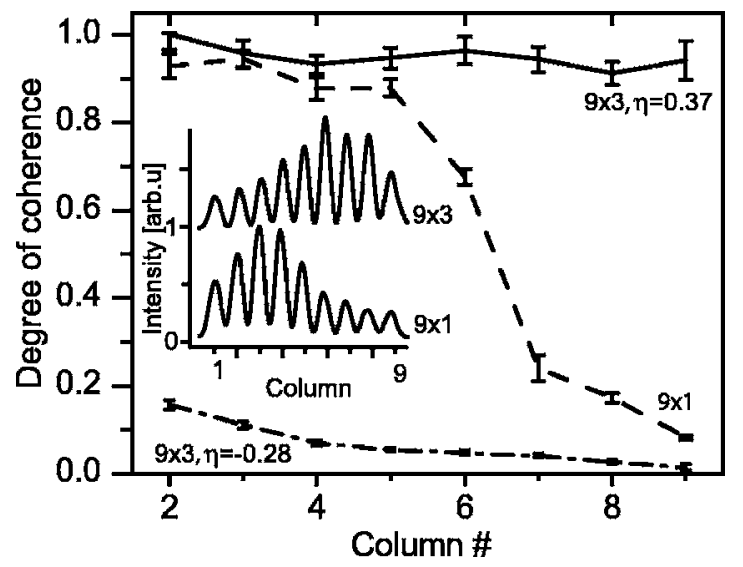

FIG. 3. Degree of spatial coherence $|\gamma|$ as a function of VCSEL column separation for a $9 \times 3$ (solid, above lasing threshold, $\eta=0.37$; dashed dotted, below threshold, $\eta=-0.28)$ and a $9 \times 1$ (dashed) VCSEL array $(\eta=0.04)$. Inset: Normalized average line scans across the $9 \times 3$ (above) and the $9 \times 1$ (below) arrays.

and below the lasing threshold, respectively. Above threshold, the average absolute detuning is $\langle|\delta|\rangle=14^{\circ}$ ( $\delta$ varies between $-31^{\circ}$ and $\left.29^{\circ}\right)$, evidencing a close to out-of-phase photonic supermode throughout the array. Below the lasing threshold, the detuning is larger, $\langle|\delta|\rangle=34^{\circ}$, and varies from $-129^{\circ}$ to $24^{\circ}$ across the array. Thus, despite the fact that the mutual coherence between the array pixels is low below threshold, most of the pixels exhibit a phase difference close to either 0 or $\pi$, indicating that amplified spontaneous emission into the main out-of-phase supermode dominates.

In order to study the variation of mutual coherence across larger interelement distances, we have made similar measurements for $9 \times 3$ and $9 \times 1$ VCSEL arrays. In this case, the mutual coherence between the selected entire columns was measured by proper setting of apertures using the spatial light modulator. Figure 3 shows that the spatial coherence between the border VCSEL column and the other VCSELs columns in the $9 \times 3$ array decreases with larger column separation. The linear decay rate is $\sim 0.01$ per column separation. Below threshold, the degree of spatial coherence is much lower, as expected, but the decay rate is similar to that measured above threshold.

For the $9 \times 1$ array structure, we observe that the mutual coherence first decays at a rate similar to that of the $9 \times 3$ array, but drops much rapidly from the sixth column (see Fig. 3 ). This is consistent with studies based on near-field and far-field measurements, which show that in arrays with lower dimensionality the coherence is degraded due to strong localization of the modal near-field patterns induced by optical disorder. ${ }^{3}$ However, comparing the variation of the spatial coherence with the near-field intensity patterns (see inset in Fig. 3), we can see that mode localization is not always coexisting with coherence degradation. In fact, the near-field pattern in the 2D array is also localized in this case, but nevertheless it exhibits a high degree of coherence across the entire structure.

The decay in mutual coherence, observed for both the $1 \mathrm{D}$ and the $2 \mathrm{D}$ arrays investigated, can be due to mode lo- calization, multimode excitation, or phase fluctuations between the optical fields of the corresponding emitters in a given mode, or a combination of all these effects. For the 1D array discussed, the degradation in coherence with emitter separation is accompanied by near-field localization, which strongly suggests a correlation between the two effects. In this case, amplified spontaneous emission dominates in the low intensity regions and is uncorrelated with the lasing mode.

For the 2D arrays, localization is significantly reduced due to the higher connectivity of the photonic lattice. ${ }^{2,12}$ In this case, the degradation in coherence across the array is gradual and is not correlated with mode localization. The partial coherence in this case (as well as the slow degradation in coherence in the 1D arrays at short distances) could be explained by the contribution of amplified spontaneous emission coupled to the nonlasing modes of the array. This possible role of amplified spontaneous emission is supported by the observed decay profile of the coherence below threshold.

The deviation from perfect out-of-phase behavior of the optical field [Fig. 2(b)] suggests that another phase variation is superimposed on the photonic envelope function. This could result from a nonuniform gain distribution across the array, which would tilt the phase front of the optical field following the imaginary part of the refractive index. Such phase tilt could also be introduced by diffraction effects at the edges of the patterned mirror. Such nonplanar phase fronts differ from the perfectly planar ones that characterize the supermodes in simple coupled mode models of realindex waveguides.

In summary, we presented results of measurements of the complex degree of spatial coherence in 1D and 2D arrays of coupled VCSELs. The results reveal details of the mutual coherence between coupled elements that cannot be inferred from simple far-field measurements. This approach should be useful for characterizing the coherence features of more complex VCSEL arrays and active photonic crystals.

This work was supported by the Fonds National Suisse de la Récherche Scientifique.

${ }^{1}$ Å. Haglund, J. S. Gustavsson, J.. Vukušić, P. Modh, and A. Larsson, IEEE Photon. Technol. Lett. 16, 368 (2004).

${ }^{2}$ A. Golshani, H. Pier, E. Kapon, and M. Moser, J. Appl. Phys. 85, 2454 (1999).

${ }^{3}$ J. J. Raftery, A. J. Danner, J. C. Lee, and K. D. Choquette, Appl. Phys. Lett. 86, 201104 (2005).

${ }^{4}$ M. Orenstein, E. Kapon, J. P. Harbison, L. T. Florez, and N. G. Stoffel, Appl. Phys. Lett. 60, 1535 (1992).

${ }^{5}$ P. L. Gourley, M. E. Warren, G. R. Hadley, G. A. Vawter, T. M. Brennan, and B. E. Hammons, Appl. Phys. Lett. 58, 890 (1991).

${ }^{6}$ N. W. Carlson, V. J. Masin, M. Lurie, B. Goldstein, and G. A. Evans, Appl. Phys. Lett. 51, 643 (1987).

${ }^{7}$ G. C. Dente, Appl. Phys. Lett. 51, 9 (1987).

${ }^{8}$ G. P. Lousberg, L. D. A. Lundeberg, D. L. Boiko, and E. Kapon, Opt. Lett. 31, 990 (2006).

${ }^{9}$ D. L. Boiko, G. Guerrero, and E. Kapon, Opt. Express 12, 2597 (2004).

${ }^{10}$ J. K. Butler, D. E. Ackley, and D. Botez, Appl. Phys. Lett. 44, 293 (1984).

${ }^{11}$ E. Kapon, J. Katz, and A. Yariv, Opt. Lett. 9, 125 (1984).

${ }^{12}$ H. Pier and E. Kapon, Opt. Lett. 22, 546 (1997). 